\title{
Guimond, L. et Desmeules, A. (2019). Des ponts interculturels à la rivière Romaine. Québec : Presses de l'Université du Québec, 117 p.
}

\author{
Marie-Claude Prémont ${ }^{\mathrm{a}}$
}

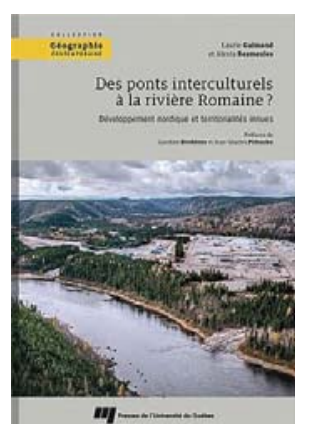

Le Québec dispose d'une longue expérience en matière de grands chantiers nordiques pour la construction de puissantes centrales hydroélectriques : depuis les centrales Bersimis-1 et Bersimis-2 des années 1950, en passant par Manic-Outardes de la fin des années 1960 jusqu'au projet du siècle de Robert Bourassa du complexe La Grande de la baie James, lancé au début de la décennie 1970.

D'autres chantiers ont également suivi, avant d'atteindre le dernier en lice, soit celui de La Romaine, sur lequel se penche l'ouvrage ici recensé. Toutefois, rappelonsnous d'abord que les deux premiers grands chantiers d'Hydro-Québec en territoire autochtone (Bersimis et Manic) se sont déployés dans l'ignorance totale de ce qu'il est aujourd'hui convenu d'appeler le territoire ancestral Nitassinan des Innus. Le chantier de la baie James a par la suite été le premier à marquer une rupture dans l'histoire hydroélectrique du Québec puisqu'un vrai coup de tonnerre judiciaire a secoué le ciel de l'inconscience et du mépris des droits autochtones, avec l'émission en novembre 1973 de l'injonction du juge Malouf, qui a forcé au silence la horde de machinerie lourde qui avait commencé à transformer le territoire cri. Même si l'injonction a été levée une semaine plus tard par la Cour d'appel du Québec, elle a néanmoins obligé Québec à s'asseoir et à négocier avec la communauté crie.

L'entente signée en 1975, intitulée la Convention de la Baie James et du Nord québécois (CBJNQ), représente le point de départ incontesté d'une ère nouvelle des relations entre le Québec et les communautés autochtones en matière de grands chantiers. Cette histoire est connue et reconnue. Personne n'oserait aujourd'hui remettre en cause les droits autochtones, depuis inscrits explicitement dans la Constitution canadienne de 1982 et, malgré tout, souvent bousculés par l'exploitation des ressources naturelles en territoire nordique.

Le plus récent grand projet hydroélectrique du Québec, soit celui de la rivière Romaine sur la Côte-Nord, représente une occasion unique de faire le point sur la place aujourd'hui réservée aux Autochtones dans le cadre d'un grand chantier hydroélectrique. Peut-on célébrer les progrès réalisés depuis l'injonction Malouf? Les auteures de cet ouvrage s'y sont penchées et actualisent la question des relations entre Autochtones et allochtones dans le cadre des grands projets hydroélectriques. Elles ne se demandent pas si Québec répond ou non aux droits autochtones. Elles se demandent, plus simplement, si le chantier permet ou non de construire, en plus des barrages et des centrales, un réel pont interculturel entre les communautés innues et non autochtones de la Minganie.

Les auteures ont su tabler sur cette occasion assez unique de La Romaine puisque le chantier permet d'observer la situation la plus contemporaine des relations issues de ce type de projet. Le chantier de La Romaine représentait en outre une occasion assez spéciale puisque l'occupation autochtone de ce territoire atteint plus du tiers de la population, caractéristique exceptionnelle au Québec, après le Nunavik.

\footnotetext{
${ }^{a}$ Professeure titulaire, ENAP
} 
Au final, se demandent les auteures, les Blancs ajustent-ils vraiment leurs projets à la réalité et à la préservation des droits autochtones, à la façon dont les Innus conçoivent et vivent leur territoire? Ou sont-ce encore plutôt les Autochtones qui doivent s'ajuster aux grands projets des Blancs? En d'autres termes, pour les Innus de la région, le projet de La Romaine représente-t-il une occasion de développement et d'actualisation de leurs relations avec le Nitassinan ou est-il plutôt un sacrifice de leur territoire?

Sur cinq chapitres et une conclusion, les deux auteures proposent en somme une analyse de l'écart qui sépare les espoirs suscités par le projet et les perceptions locales quant aux retombées réelles sur le territoire directement touché par le grand chantier de La Romaine. La particularité de l'analyse des auteures tient au fait qu'elles s'attardent au territoire vécu de la Minganie, ce qui place davantage en relation non pas le Québec et la communauté innue dans son ensemble, mais bien les personnes mêmes qui habitent ou séjournent sur le territoire : d'une part, les quatre communautés innues touchées (Nutashkuan, Unamem Shipu, Pakua Shipi et Ekuanitshit) et, d'autre part, les communautés allochtones de la région (Rivière-au-Tonnerre, Rivière-Saint-Jean, Longue-Pointe-de-Mingan, Havre-Saint-Pierre, Baie-Johan-Beetz, Aguanish et Natashquan).

Par contre, l'ouvrage révèle que les non-Autochtones sondés sont essentiellement les travailleurs du chantier (qui proviennent majoritairement de l'extérieur de la région), mais aussi des « acteurs clés » de la région, disentelles, mais dont on ne connait pas précisément le rattachement. Sont-ce des maires, des préfets, des dirigeants d'entreprise? Le chantier de La Romaine représente certes une occasion d'exception pour jauger l'interaction entre les Innus et les autres travailleurs. C'est pourquoi, pour les auteures, il forme le site privilégié de l'analyse, qui s’appuie sur environ 60 entrevues approfondies réalisées sur le terrain.

L'hypothèse explorée par les auteures pose d'emblée la difficulté d'établir des ponts entre Autochtones et nonAutochtones. En dépit de tous les progrès qui ont pu marquer les relations entre les deux groupes, les auteures observent qu'ils continuent à vivre des vies parallèles, même au sein du chantier, où les deux groupes sont pourtant convoqués. Elles concluent à une quasi-absence de relations interculturelles significatives. Les cliques se forment par classe de métier, mais aussi par région d'origine des travailleurs; deux caractéristiques systémiques opèrent à creuser un fossé plutôt qu’à établir des ponts.

Les travailleurs innus forment en grande partie une main-d'œuvre invisible et au bas de l'échelle. Sur 144 Innus engagés par la Société des entreprises innues d'Ekuanitshit (SEIE), 108 sont affectés à la cafétéria et à la conciergerie, alors que 12 sont à l'entretien technique et 15 à la sécurité. Les auteures donnent quelques exemples, rares, d'Innus qui occupent un poste plus élevé, comme celui d'opérateur de machinerie lourde. De plus, les Innus sont confinés à des dortoirs distincts, contredisant de manière forte toute velléité de rapprochement des communautés. Les auteures rapportent également la perception répandue du phénomène de l'Innu-pancarte, avec la création de sociétés innues, souvent partenaires d'entreprises allochtones (dont la compagnie française Sodexo), pour bien faire paraitre l'entreprise qui porte un nom innu.

Les clés de lecture des différents chapitres ne sont pas toujours bien distinguées, de sorte que certaines observations ou analyses qui auraient pu être mieux explorées sont plutôt parsemées à travers l'ouvrage. C'est notamment le cas du rôle joué par les ententes conclues entre Hydro-Québec et les différentes communautés, phénomène central qui a pris son envol depuis la CBJNQ afin de structurer les relations entre les communautés autochtones et les entreprises d'exploitation des richesses naturelles, dont Hydro-Québec. En effet, sans faire l'objet d'une analyse détaillée, les auteures parsèment leurs propos ici et là dans l'ouvrage du fait que tant les communautés autochtones qu'allochtones ont signé avec Hydro-Québec, derrière des portes closes, des ententes sur les répercussions et avantages (ERA). Elles soulignent que toutes les communautés ont signé de telles ententes : une pour la municipalité régionale de comté (MRC) de la Minganie et trois ERA pour les quatre communautés innues du territoire. La négociation de ces ententes, en secret et communauté par communauté, a suscité des tensions interculturelles, contraires au rapprochement interculturel. L'ERA est dès lors pointée comme un instrument de division entre les communautés. On peut regretter que les auteures n'aient pas profité de l'occasion pour mieux documenter le fonctionnement et les effets précis des ERA pour répondre à leur enquête sur la construction de ponts interculturels. 
Elles soulignent pourtant bien le dilemme auquel font face les Innus, qui n'ont d'autre choix devant la puissance d'Hydro-Québec que d'accepter de négocier, ce qui signifie du coup de mettre fin au combat. Il est intéressant de noter que la dernière communauté innue à avoir signé une telle entente est celle qui est la plus touchée par le projet, soit la communauté d'Ekuanitshit. La réaction des Blancs frise ensuite le sarcasme en remettant en cause l'attachement réel des Innus au Nitassinan puisqu'ils acceptent de le sacrifier en échange des bénéfices accordés par l'ERA.

Pourtant, sur le territoire, les auteures font certaines observations qui permettent d'établir une certaine communauté d'intérêt entre Autochtones et non-Autochtones en s'attardant justement aux habitants du territoire, et non aux travailleurs du chantier. En effet, comme les Innus, les travailleurs de la Minganie n'arrivent pas à tirer leur épingle du jeu dans la promesse des emplois générés par le grand chantier. La plupart des contrats de sous-traitance sont accordés à des entreprises hors région qui recrutent leurs employés habituels, au détriment des travailleurs de la région. L'ouvrage rapporte que les travailleurs de la Minganie ne dépassent pas $15 \%$ des travailleurs du chantier, tandis que les Innus ne sont que de $11 \%$. Même si le texte n'est pas clair à ce sujet, il faut croire que les $11 \%$ d'Innus ne sont pas comptabilisés dans les $15 \%$ de travailleurs de la Minganie. Comme les Innus, les travailleurs de la Minganie occupent aussi surtout des emplois au bas de l'échelle, ce qui permettrait de dire que les seuls ponts interculturels créés par le chantier de La Romaine se limitent à la communauté d'intérêts des plus démunis du chantier, où Autochtones et non-Autochtones du territoire sont maintenus au bas de l'échelle des emplois.

Le chantier de La Romaine n'aura été au fond qu'une simple étincelle, pour reprendre l'expression des auteures, afin de sensibiliser à l'importance de construire des ponts interculturels. La place limitée des Innus sur le chantier est un reflet de leur place politico-institutionnelle restreinte, tout comme celle des Minganiens, pourrait-on ajouter. En ce, Hydro-Québec ne peut être seule mise au banc des accusés puisque l'atteinte d'une meilleure équité et justice entre communautés est une mission qui interpelle toutes nos institutions et en tout temps. Les grands projets hydroélectriques ne peuvent être que le révélateur de nos déficiences institutionnelles, et non les seuls réparateurs. Le chantier de La Romaine le démontre, encore une fois. 